\title{
Seabed Tracking of an Autonomous Underwater Vehicle with Nonlinear Output Regulation
}

\author{
A. Adhami-Mirhosseini, A. Pedro Aguiar and M. J. Yazdanpanah
}

\begin{abstract}
This paper proposes a new control algorithm for automatic seabed tracking performed by a low cost autonomous underwater vehicle subject to the fact that the seabed profile is not known in advance. The control design amounts to cast the problem in the framework of nonlinear output regulation theory combined with pseudo-spectral methods to approximate the solutions of the regulator equations. To this end, the seabed tracking problem is re-formulated as a trajectory tracking problem and the reference signal is constructed using Fourier series to approximate the seabed profiles. An interesting feature of this approach is that the combination of the Fourier series with output regulation problem allows to bypass the need to compute explicitly the Fourier coefficients. Stability analysis and simulation results with real seabed data show the effectiveness of the proposed controller.
\end{abstract}

\section{INTRODUCTION}

In the last decade there has been an increasing number of scientific (and military) applications that require unmanned underwater vehicles to execute traverses at a constant altitude from the sea bottom. This task is called seabed tracking or bottom following in the literature. Its solution is strongly dependent by the following fact: $i$ ) there is a map of the operating environment, or in the other case ii) the area is unknown. This paper is concerned with the latter problem where the speed profile is not known in advance. We propose a solution to the problem of seabed tracking controller design for a small, low cost, autonomous underwater vehicle (AUV). We consider that the sensors are limited and, in particular, that the altitude measurement is carried out by a single beam acoustic altimeter sensor. The proposed solution is to be applied to the control of the prototype Medusa AUV, operated by the Instituto Superior Tecnico of Lisbon, Portugal.

One of the first works on the seabed tracking using unmanned vehicles can be traced to the work described in [1] where Proportional Integrator (PI) controllers are proposed. In [2] a Lyapunov based controller for a Remotely Operated Vehicle (ROV) is developed that take into account disturbances and uncertainties. The control law uses the current distance from seabed and the seabed slope. These

A. Adhami-Mirhosseini and M.J. Yazdanpanah are with the Control and Intelligent Processing Center of Excellence, University of Tehran, P.O. Box 14395/515 Tehran, Iran. aras . adhami dece.ut . ac. ir and yazdandut.ac.ir

A.P. Aguiar is with the Lab. of Robotics and Systems in Engineering and Science (LARSyS), Inst. Superior Tecnico (IST), Technical University of Lisbon, Av. Rovisco Pais, 1049-001 Lisboa, Portugal. pedrodisr.ist.utl.pt

This work was supported by projects CONAV/FCT-PT (PTDC/EEACRO/113820/2009), Co3-AUVs (EU FP7 no.231378), FCT-ISR/IST plurianual funding program, and the CMU-Portugal program. two quantities are estimated using the measurements given by two echo sounders.

Another method proposed in [3] for seabed tracking takes into account the terrain characteristics ahead of the vehicle, that are measured by two echo sounders. The main idea amounts to formulate the problem as a discrete time path following control task, where a conveniently defined state error in the space model of the plant is augmented with bathymetric preview data.

In this paper, we exploit the framework of output regulation theory and combine with it pseudo-spectral methods to approximate the solutions of the regulator equations. To this effect, we re-formulate the seabed tracking problem as a trajectory tracking problem and construct the reference signal using Fourier series to approximate the seabed profiles. It turns out that with this approach the Fourier coefficients are not needed to be computed explicitly.

From a practical point of view, the main contribution of the paper compared with other methods applied to seabed tracking is the fact that we only need one simple sensor that provides the distance of the vehicle to the seabed, and also that the seabed profile is not needed to know in advance. Furthermore, there is no need to obtain measurements of the heave velocity (that is, the vertical linear velocity).

In addition, we consider the practical situation that there exist inner-loop tracking controllers for the linear surge velocity $u$ and pitch angular velocity $q$ and take their dynamics into account in the control design.

The paper is organized as follows. Section II describes the nonlinear model for the vertical plane dynamics of the Medusa AUV and formulates the problem of seabed tracking. Section III introduces briefly the output regulation problem and Section IV states the controller design procedure. The stability analysis is discussed in Section $\mathrm{V}$ and in Section VI the performance of the control algorithm is evaluated using computer simulations and real seabed data. Finally, Section VII contains some concluding remarks and discusses problems that warrant further research.

\section{CONTROL PROBLEM FORMULATION}

This section describes the AUV equations of motion in vertical plane and formulates the seabed tracking problem.

\section{A. AUV modelling}

Following the general practice to describe the motion of an AUV, we resort to an earth-fixed coordinate frame $\{U\}$ and a body-fixed coordinate frame $\{B\}$. The complete kinematic and dynamic equations of motion is described in e.g. [4]. In 
the vertical plane by neglecting the motion in the sway, roll and yaw, the kinematic equations take the form

$$
\begin{aligned}
& \dot{x}=u \cos \theta+w \sin \theta \\
& \dot{z}=-u \sin \theta+w \cos \theta \\
& \dot{\theta}=q
\end{aligned}
$$

where $u, w$ and $q$ are the linear and angular velocities of the vehicle, respectively, in surge $(x)$, heave $(z)$ and pitch $(\theta)$ direction of the body-fixed coordinates. The Cartesian coordinates of the vehicle's center of mass is denoted by $x$ and $y$, and $\theta$ is the pitch angle. The dynamic equations of the vertical motion when there in no actuated force in $Z_{B}$ direction (that is, the vehicle is underactuated) are given by

$$
\begin{aligned}
& m_{u} \dot{u}+m_{w} w q+d_{u} u=\tau_{u} \\
& m_{w} \dot{w}-m_{u} u q+d_{w} w=0 \\
& m_{q} \dot{q}+m_{u w} u w+d_{q} q=\tau_{q}
\end{aligned}
$$

where $m_{u}=m-X_{\dot{u}}, m_{w}=m-Z_{\dot{w}}, m_{q}=I_{y}-M_{\dot{q}}$ and $m_{u w}=m_{u}-m_{w}$ are mass and hydrodynamic added mass terms and $d_{u}=-X_{u}-X_{u|u|}|u|, d_{w}=-Z_{w}-Z_{w|w|}|w|$ and $d_{q}=-M_{q}-M_{q|q|}|q|$ are hydrodynamic damping effects. These scalar parameters are listed in the simulation section for the Medusa AUV. The symbols $\tau_{u}$ and $\tau_{q}$ denote the external force in surge direction and the external torque around the $y$-axis of the vehicle, respectively.

We consider the practical situation that there exist innerloop controllers in charge of tracking reference signals in $u$ and $q$. For simplicity, we assume that these autopilots controllers can be locally characterized by a first order stable dynamics.

\section{B. Problem formulation}

The goal of seabed tracking is roughly speaking to keep constant the vertical distance between the AUV and the seabed. Let $z_{s}=z_{s}(x)$ be the seabed depth profile in function of the position $x$.

The problem of seabed tracking can be stated as follow: Consider the AUV vertical model (1) and (2) together with measurements on the depth $z$ and altitude $h$ from the seabed, i.e. $z_{s}=z+h$. Derive feedback control laws for the surge reference velocity $u_{r}$ and pitch angular reference velocity $q_{r}$ to drive the vehicle to move along an $X_{B}$ direction with a desired horizontal velocity $V_{x}$ at a constant height $h_{d}$ from the seabed. In a more practical case, we are looking for a controller that keeps the altitude $z_{s}-z$ around a constant $h_{d}$ value.

\section{NONLINEAR OUTPUT REGULATION}

The framework of nonlinear output regulation as a tool for reference tracking and/or disturbance rejection has been received considerable attention in the last decades [5], [6] and [7]. In what follows we briefly point out the basics of this methodology. For convenience in this section we will adopt some of the conventional notation in the output regulation theory, which is not consistent and should not be associated to the notation introduced in the previous section. Consider the nonlinear system

$$
\left\{\begin{array}{l}
\dot{x}=f(x, u, v) \\
y_{m}=h_{m}(x, v) \\
e=h(x, v)
\end{array}\right.
$$

where $x \in \mathbb{R}^{n}$ is the state of the system, $u \in \mathbb{R}^{m}$, $y_{m} \in \mathbb{R}^{p_{m}}$, and $e \in \mathbb{R}^{p_{e}}$ are control input, measurement output and regulated output, respectively. The functions $f$, $h_{m}$ and $h$ are Lipschitz functions in all arguments and vanishing at the origin. The signal $v \in \mathbb{R}^{q}$ is considered as a reference/disturbance signal. This signal is generated by the exo-system

$$
\dot{v}=s(v)
$$

The problem of local output regulation consists in finding a control law

$$
\begin{aligned}
& \dot{\eta}=\sigma\left(\eta, y_{m}\right) \\
& u=k\left(\eta, y_{m}\right)
\end{aligned}
$$

such that, there exists a neighborhood around the origin that for any closed-loop trajectory that starts from it, achieves asymptotically zero regulated output with bounded states.

Depending on the measurement output, two type of controllers may be used. When all the plant and exo-system states are available, i.e., $y_{m}=(x, v)$, full information static feedback controller by setting $\eta=0$ in (5) is enough to find a possible solution of the output regulation problem. Dynamic output feedback controller in general is needed when all states are not available for feedback.

There are several approaches to precisely define the nonlinear output regulation problem and derive necessary and sufficient conditions for solvability of the defined problem. The first approach was introduced by [8] and [9] in two seperate works. This approach is based on the center manifold theorem and applicable to systems with $C^{2}$ vector fields. In [7], the authors describe another approach based on the convergent dynamics concept. Since the AUV motion dynamics (1) and (2) contains $C^{1}$ functions (and not $C^{2}$ ), we focus on the second approach that is called uniform output regulation [7]. To find the precise definition of the problem and related concepts, see [7] (chapter 3). A theorem stated in [7] provides the necessary and sufficient condition for solvability of the local uniform output regulation problem for system (3) with exo-system (4). Briefly, the main condition required is that there should exist mappings $x=\pi(v)$ and $u=\alpha(v)$ with $\pi(0)=0$ and $\alpha(0)=0$ that satisfy the regulator equation

$$
\begin{aligned}
& \frac{\partial \pi(v)}{\partial v} s(v)=f(\pi(v), \alpha(v), v) \\
& h(\pi(v), v)=0
\end{aligned}
$$

in some invariant neighborhood $v=0$.

The regulator equation (6) plays a main role in the output regulation problem. However, in general this set of $n$ partial differential equations and $m_{e}$ algebraic equations has no closed solution. 


\section{Controller Design}

In this section we cast the seabed tracking problem in the output regulation setup and derive an approximation of the solution of the regulator equations using Pseudospectral approximation methods. A full information and error feedback controllers are then obtained.

\section{A. Nonlinear output regulation for seabed tracking problem}

To formulate the seabed tracking problem in the nonlinear output regulation format we first have to define the reference signal that needs to be generated by a neutrally stable exosystem. To this effect, we consider that the seabed profile is a finite combination of $N$ sinusoidal functions with known frequencies $\Omega_{i}$ and unknown amplitudes $A_{i}$ and phases $\varphi_{i}$, i.e.,

$$
z_{s}(x)=A_{0}+\sum_{i=1}^{N} A_{i} \sin \left(\Omega_{i} x+\varphi_{i}\right)
$$

To represent (7) as a function of time, we also consider that there exists a speed controller that regulate the surge velocity $u$ to a given reference value $u_{r}(t)$. The reference signal is computed such that the horizontal velocity is constant to some desired value $V_{x}$. In this case, from (1) the corresponding reference signal is given by

$$
u_{r}=\frac{V_{x}-w \sin \theta}{\cos \theta}
$$

Note that we assume that the pitch angle of the vehicle is not close to the singular points $(2 k \pm 1) \pi / 2$, which in practice for this type of marine vehicles is a reasonable assumption. Also, later, $u_{r}$ will be redefined so that we do not need to measure the heave velocity $w$.

From (8), it follows now that when $u=u_{r}$ we have $\dot{x}=$ $V_{x}$ and therefore $x=V_{x}\left(t-t_{0}\right)+x\left(t_{0}\right)$. Without loss of generality set $t_{0}=0$ and $x(0)=0$. By this relation between time and horizontal position, the seabed profile (7) can be rewritten as a time dependent signal

$$
z_{s}(t)=A_{0}+\sum_{i=1}^{N} A_{i} \sin \left(\Omega_{i} V_{x} t+\varphi_{i}\right)
$$

This reference signal can be produced by the following autonomous neutrally stable system recalled exo-system

$$
\begin{aligned}
& \dot{v}=S v \\
& S=V_{x} \operatorname{Diag}\left(0,\left[\begin{array}{c}
0, \Omega_{1} \\
-\Omega_{1}, 0
\end{array}\right], \because,\left[\begin{array}{c}
0, \Omega_{N} \\
-\Omega_{N}, 0
\end{array}\right]\right)
\end{aligned}
$$

where $v=\left[v_{0}, v_{1,1}, v_{2,1}, \cdots, v_{1, N}, v_{2, N}\right]^{T} \in \mathbb{R}^{2 N+1}$ is the state vector. The output $y_{s}$ of the exo-system is defined as

$$
y_{s}=v_{0}+v_{1,1}+v_{1,2}+\ldots+v_{1, N}
$$

By setting the initial conditions to the exo-system as $v_{0}(0)=$ $A_{0}, v_{1, i}(0)=A_{i} \sin \left(\varphi_{i}\right)$ and $v_{2, i}(0)=A_{i} \cos \left(\varphi_{i}\right)$ for $i=$ $1, \ldots, N$, the reference signal (9) is equal to the output of the exo-system, i.e. $z_{s}(t)=y_{s}(t)$ for all $t \in \mathbb{R}^{+}$.

The AUV equations with $u=u_{r}$ is reduced to

$$
\dot{x}_{p}=f_{p}\left(x_{p}, q\right)
$$

where $x_{p}=[z, w, \theta]^{T}$ and

$$
\begin{aligned}
& f_{p}\left(x_{p}, q\right)= \\
& \quad\left[\begin{array}{c}
V_{x} \tan \theta+\frac{w}{\cos \theta} \\
\frac{Z_{w}}{m_{w}} w+\frac{Z_{w|w|}}{m_{w}} w|w|+\frac{m_{u}}{m_{w}}\left(\frac{V_{x}}{\cos \theta}-w \tan \theta\right) q \\
q
\end{array}\right]
\end{aligned}
$$

Now, the seabed tracking problem is equivalent to the output regulation problem with plant model (11), exo-system (10) and error $e=y_{s}-z-h_{d}$. To simplify the notation in design procedure, we replace $z+h_{d}$ by $z$. Thus, hereafter the output to be regulated is changed to $e=y_{s}-z$. Note that, this transformation in $z$ does not change the vehicle dynamics of motion in the vertical plane, (1) and (2).

\section{B. Full-information feedback output regulator design}

To design the output regulator, we first start with the full information feedback case. Following the output regulation framework, [5] and [8], we are looking for a controller of the form $q=q(z, w, \theta, v)$ such that for sufficiently small initial conditions of the plant model and the exo-system, the error output is annihilated by time, i.e. $\lim _{t \rightarrow \infty} y_{s}(t)-z(t)=0$

One particular choice for the controller is $q=q_{r}=$ $\alpha_{q}(v)+K\left(x_{p}-\pi(v)\right)$, where $\alpha_{q}(v)$ and $\pi(v)=$ $\left[\pi_{z}(v), \pi_{w}(v), \pi_{\theta}(v)\right]^{T}$ are mappings such that $\alpha_{q}(0)=0$ and $\pi(0)=0$ and satisfy the regulator equations

$$
\begin{aligned}
\frac{\partial \pi_{z}}{\partial v} S v= & -V_{x} \tan \pi_{\theta}+\frac{\pi_{w}}{\cos \pi_{\theta}} \\
\frac{\partial \pi_{w}}{\partial v} S v= & \frac{Z_{w}}{m_{w}} \pi_{w}+\frac{Z_{w|w|} m_{w}}{m_{w}}\left|\pi_{w}\right| \\
& +\frac{m_{u}}{m_{w}}\left(\frac{V_{x}}{\cos \pi_{\theta}}-w \tan \pi_{\theta}\right) \alpha_{q} \\
\frac{\partial \pi_{\theta}}{\partial v} S v= & \alpha_{q} \\
0 & v_{0}+v_{1,1}+v_{1,2}+\ldots+v_{1, N}-\pi_{z}
\end{aligned}
$$

By solving these equations, the mappings are found. To complete the controller, the matrix gain $K$ should be computed such that it stabilizes the linear part of the reduced plant model (11) at the origin characterized by

$$
A_{K} \triangleq \frac{\partial f_{p}}{\partial x_{p}}(0,0)+\frac{\partial f_{p}}{\partial q}(0,0) K
$$

Some remarks about this controller worth to be mentioned:

- In general, it is difficult to find the exact solution to the regulator equations (12). Later we propose a method to approximate the solution.

- Since the linear part of the reduced plant model (11) is controllable, it is possible to find a stabilizer gain $K$. One way is to use the linear quadratic regulator theory with properly chosen state and control weighted matrices.

- To use the proposed controller, we need measurements on all the states of the vehicle and exo-system. Thus, designing an output regulator with output feedback is necessary in practice.

\section{Output feedback output regulator design}

To design an output regulator with output feedback, we need to find a dynamic controller of the form

$$
\begin{aligned}
& \dot{\eta}=\sigma\left(\eta, y_{m}\right) \\
& q_{r}=k\left(\eta, y_{m}\right)
\end{aligned}
$$


where $y_{m}$ satisfies the output equation

$$
y_{m}=\left[\begin{array}{c}
z \\
z_{s}
\end{array}\right]=C_{m} x_{p}+F_{m} v
$$

with $C_{m}=\left[\begin{array}{lll}1 & 0 & 0 \\ 0 & 0 & 0\end{array}\right]$ and $F_{m}=\left[\begin{array}{cccc}0 & & \\ 1 & 1 & 0 \cdots 1 & 0\end{array}\right]$. Note that in (14), $y_{m}$ is obtained from the data given by the sensors that measure the depth $z$ and the altitude $h$ (where $z_{s}=z+h$ ). In the output regulator framework one can find several approaches to design the dynamic controller, see e.g., [5], [10] and [6]. In this paper we apply the method described in [6], that consists in using the state feedback control law derived in Section II.B but replacing the state by its estimate. The dynamic controller generates $\eta_{1}$ and $\eta_{2}$ to estimate $x_{p}$ and $v$, respectively, and takes the form

$$
\begin{aligned}
& \left.\dot{\eta}_{1}=f_{p}\left(\eta_{1}, q_{r}\right)\right)-L_{1}\left(y_{m}-C_{m} \eta_{1}\right) \\
& \dot{\eta}_{2}=S \eta_{2}-L_{2}\left(y_{m}-F_{m} \eta_{2}\right) \\
& q_{r}=\alpha_{q}\left(\eta_{2}\right)+K\left(\eta_{1}-\pi\left(\eta_{2}\right)\right)
\end{aligned}
$$

where $\eta_{1} \in \mathbb{R}^{3}$ and $\eta_{2} \in \mathbb{R}^{2 N+1}$ are controller states, $\alpha_{q}($. and $\pi($.$) are the same mappings as in the full-information$ feedback output regulation that are obtained from the regulator equation (12), and $L_{1}$ and $L_{2}$ are matrix gains such that the following matrix

$$
A_{L} \triangleq\left[\begin{array}{cc}
A_{p} & 0 \\
0 & S
\end{array}\right]+\left[\begin{array}{l}
L_{1} \\
L_{2}
\end{array}\right]\left[\begin{array}{ll}
C_{m} & F_{m}
\end{array}\right]
$$

with $A_{p}=\frac{\partial f_{p}}{\partial x_{p}}(0,0)$ is Hurwitz. Some remarks on the output regulator by measurement feedback are now discussed.

- The dynamic controller (16) consists of an extended Kalman filter for the reduced plant model (11) and a Kalman filter for the exo-system (10). These state estimates can be easily shown to converge to the true values by the asymptotic stability of the origin of the estimation errors $\hat{x}_{p}=x_{p}-\eta_{1}$ and $\hat{v}=v-\eta_{2}$ dynamics.

- By the result of the previous remark, the reference signal (8) for linear velocity can be modified to use the estimated states $\eta_{1}=\left[\eta_{z}, \eta_{w}, \eta_{\theta}\right]^{T}$, that is,

$$
u_{r}=\frac{V_{x}-\eta_{w} \sin \eta_{\theta}}{\cos \eta_{\theta}}
$$

- The dynamic feedback control law (16) together with the velocity controller (18) achieve seabed tracking requiring only to know the value $N$ (number of sinusoidal functions generated by the exo-system to obtain the reference signal that approximates the seabed profile), the frequencies $\Omega_{i}, i=1, \ldots, N$ and the measurements of depth $z$ and altitude $h=z_{s}-z$.

\section{Approximate Output feedback output regulator}

As mentioned, one problem in the output regulator framework is the difficulty to find a closed solution to the regulator equation (12). Approximation is an alternative way to overcome this difficulty. For that effect, one of the oldest method is to use truncated Taylor series expansion for approximation of the solutions of the regulator equation. Although, Taylor series is an easy method in computation and complexity, it is not good in accuracy in some problems. In our case, since the regulator equation (12) is not smooth, Taylor series method fails to find an approximate solution to it. One way is to approximate the nonsmooth function $\pi_{w}\left|\pi_{w}\right|$ by a smooth function, but still Taylor series expansion does not provide a good approximate solution when $v$ is not so close to the origin.

To solve the problem of convergency and accuracy in distant point, an approximation method which focuses on a predefine interval is proposed. Pseudo-spectral method is a good choice, because it offers high accuracy with fast convergence in approximation order, close solution and reasonable computational complexity. In the following, we briefly describe the pseudo-spectral method for the output regulation problem.

The main idea in the pseudo-spectral method for approximation of functions, is to compute the function at some collocation points and then linearly combine the basis polynomials based on the computed values to approximate the function. These basis are orthogonal set of polynomials with respect to an inner product in the desired space.In our case, we use Chebyshev polynomials and the unknown function is the solution to the regulator equation. Assume the general regulator equation (6) and suppose that we are concerned to about the solution in a rectangular subspace $\mathcal{I} \subset \mathbb{R}^{q}$. Based on the Chebyshev polynomials, we set collocation points $\xi_{i} \in \mathcal{I}$ for $i=1, \ldots, m_{a}$. The number of collocation points $m_{a}$ is related to the approximation order that is set and depends on the required accuracy. Now, the solution to the regulator equations should be computed in the collocation points i.e. $\pi\left(\xi_{i}\right)$ and $\alpha\left(\xi_{i}\right)$ for $i=1, \ldots, m_{a}$. This is done by solving the regulator equation in these points. Finding unknown mappings in collocation points from the regulator equation result in a set of nonlinear algebraic equations. After solving this set of equations, usually by a simple numerical method, approximate solution to the regulator equation $\pi(v)$ and $\alpha(v)$ is obtained for $v \in \mathcal{I}$ as

$$
\begin{aligned}
& \pi(v) \approx \sum_{i=1}^{m_{a}} \pi\left(\xi_{i}\right) l_{i}(v) \\
& \alpha(v) \approx \sum_{i=1}^{m_{a}} \alpha\left(\xi_{i}\right) l_{i}(v)
\end{aligned}
$$

where $l_{i}(),. i=1, \ldots, m_{a}$ are Lagrange polynomials with respect to the selected collocation points.For further details of the pseudo-spectral method in general see [11] and [12].

\section{E. General seabed profile}

Until this point, we have considered the case that the seabed profiles can only take a shape composed by a finite summation of sinusoidal functions with different known frequencies. To deal with general seabed profiles, we use the fact that each periodic function can be approximated by harmonic sinusoidal functions. Fourier series expansion is a tool that does this approximation, [13].

Suppose that $z_{s}(x)$ is a real seabed that we would like the vehicle to track and let $x \in\left[0, T_{x}\right]$ be a given interval. To generate the reference signal using Fourier series, we assume that $z_{s}(x)$ is periodic with period $T_{x}$. Now we can 


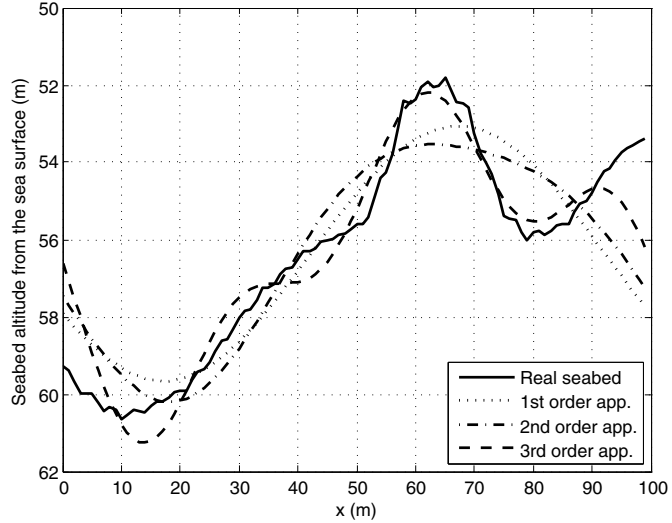

Fig. 1. Fourier series seabed approximation by different approximation orders.

approximate $z_{s}$ by $\hat{z}_{s}$ as

$$
\hat{z}_{s}=a_{0}+\sum_{m=1}^{M} A_{m} \sin \left(m \Omega_{0} x+\varphi_{m}\right)
$$

where $M$ is the seabed approximation order and $\Omega_{0}=\frac{2 \pi}{T_{x}}$ is the main frequency of approximate signal that only depends on the predefined length of the tracking interval.

This approximation of the seabed is in the format of (7) by setting $N=M$ and $\Omega_{i}=i \Omega_{0}$, and therefore it can be generated by the exo-system (10). By increasing the seabed approximation order $M, \hat{z}_{s}$ converge to $z_{s}$. The rate of convergence depends on the smoothness of the seabed profile, [13]. Fig. 1 shows a real volcanic seabed and its approximations with different orders. Note that the resulting controller using this exo-system enable to drive the vehicle to move along an $M$ th-order Fourier approximation of the real seabed. Furthermore, we do not need to compute the Fourier coefficients to generate the reference signal. The exo-system only depends on the parameters $M$ and $T_{x}$.

\section{Stability ANALYSis}

To show the stability and performance achievement of the closed-loop system, we consider the output feedback controller with sinusoidal seabed profiles and exact solution of the regulator equations.

Theorem 1: Consider the reduced model of the AUV motion in the vertical plane (11) and seabed profiles of the type (7). Consider the outer-loop output feedback controller

$$
\begin{aligned}
& \dot{\eta}_{1}=f_{p}\left(\eta_{1}, k\left(\eta_{1}, \eta_{2}\right)\right)-L_{1}\left(y_{m}-C_{m} \eta_{1}\right) \\
& \dot{\eta}_{2}=S \eta_{2}-L_{2}\left(y_{m}-F_{m} \eta_{2}\right) \\
& q_{r}=\alpha_{q}\left(\eta_{2}\right)+K\left(\eta_{1}-\pi\left(\eta_{2}\right)\right)
\end{aligned}
$$

where $K, L_{1}$, and $L_{2}$ are selected so that (13) and (17) are Hurwitz matrices, $\pi$ and $\alpha_{q}$ are the solution to the regulator equations (12) in some neighborhood $\mathcal{W} \subset \mathbb{R}^{2 N+1}$ of the origin.

Then, for sufficiently small initial condition the closedloop system has bounded states and the vehicle altitude converges to a desired constant distance $h_{d}$ from the seabed, i.e. $\lim _{t \rightarrow \infty} z_{s}(t)-z(t)=h_{d}$.
TABLE I

Medusa AUV PARAMETERS

\begin{tabular}{ccc} 
Parameter description & Symbol & Value \\
\hline \hline Vehicle mass $(\mathrm{kg})$ & $\mathrm{m}$ & 30 \\
Added masses $(\mathrm{kg})$ & $\left(X_{\dot{u}}, Z_{\dot{w}}\right)$ & $(-2.2,-4.0)$ \\
Damping $(\mathrm{kg} / \mathrm{s}, \mathrm{kg} / \mathrm{m})$ & $\left(Z_{w}, Z_{w|w|}\right)$ & $(-3,-12.4)$
\end{tabular}

TABLE II

CONSTANT PARAMETERS IN THE SIMULATIONS

\begin{tabular}{ccccc} 
Parameter description & Symbol & \multicolumn{3}{c}{ Value } \\
\hline \hline Horizontal velocity $(\mathrm{m} / \mathrm{s})$ & $V_{x}$ & 0.1 \\
Desired altitude $(\mathrm{m})$ & $h_{d}$ & 5 & 5 \\
Controller gain & $K$ & {$\left[\begin{array}{ccc}22.36 & 147.32 & -24.91\end{array}\right]$} \\
Observer gain & $L_{1}$ & {$\left[\begin{array}{ccc}-3.97 & -2.56 & 3.16 \\
0 & 0 & 0\end{array}\right]^{T}$} \\
Observer gain & $L_{2}$ & {$\left[\begin{array}{ccc}0 & 0 & 0 \\
-0.42 & -1.35 & -1\end{array}\right]^{T}$}
\end{tabular}

The next theorem takes into consideration the dynamics of the inner-loop tracking controllers.

Theorem 2: Consider the AUV motion in the vertical plane (1) and (2) together with the inner-loop tracking controllers, the seabed profile of the type (7), and the dynamic feedback controller (21) where $K, L_{1}$, and $L_{2}$ are selected so that (13) and (17) are Hurwitz matrices, $\pi$ and $\alpha_{q}$ are the solution to the regulator equations (12) in some neighborhood $\mathcal{W} \subset \mathbb{R}^{2 N+1}$ of the origin.

Then, for sufficiently small initial condition the closedloop system has bounded states and the vehicle altitude converges to a desired constant distance $h_{d}$ from the seabed, i.e. $\lim _{t \rightarrow \infty} z_{s}(t)-z(t)=h_{d}$.

\section{Simulation RESUlts}

To show the effectiveness of the output regulator with measurement feedback, we performed computer simulations using the Medusa AUV model. Table I shows the values of the parameters. Two set of simulations illustrate the proposed output feedback controller performance. The controller parameters and simulation conditions fixed for all simulations are listed in Table II. The first set of simulations is concerned about the tracking of the sinusoidal seabed $z_{s}=48+5 \sin \left(0.2 x+\frac{\pi}{4}\right)$ with a desired vertical distance $h_{d}$. Note that the vehicle does not know in advance the seabed profile. The initial condition of the dynamic output feedback controller (16) is set to $\eta_{1}(0)=[0,0,0]^{T}$ and $\eta_{2}(0)=[0,0,30]^{T}$. Fig. 2 shows the vehicle trajectories starting from four different initial conditions

$$
\begin{aligned}
& {[x(0), z(0), w(0), \theta(0)]=[0,20,-1,-\pi / 4]} \\
& {[x(0), z(0), w(0), \theta(0)]=[0,30,1, \pi / 4]} \\
& {[x(0), z(0), w(0), \theta(0)]=[0,40,0, \pi / 6]} \\
& {[x(0), z(0), w(0), \theta(0)]=[0,50,-1,-\pi / 6]}
\end{aligned}
$$

These simulations reveal that, although the proposed controller only guarantees local stability, it is not much sensitive to the initial condition. Fig. 3 shows the vehicle trajectory described by the vehicle when it is required to track a real 


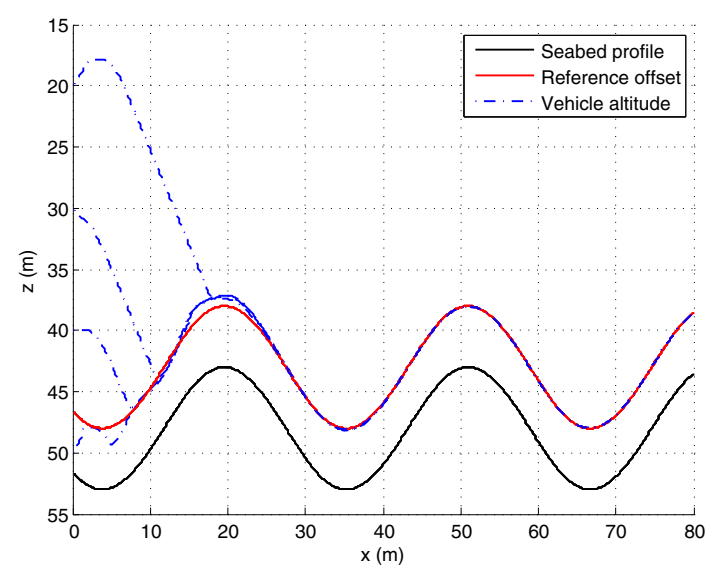

Fig. 2. Trajectories described by the vehicle with output feedback output regulator for a sinusoid seabed profile.

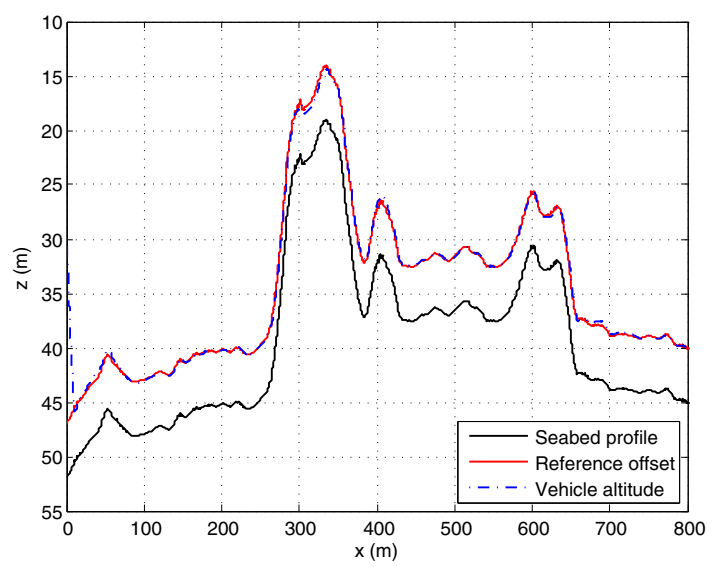

Fig. 3. Trajectory described by the vehicle with output feedback output regulator for a real seabed profile.

seabed. This seabed profile belongs to the volcanic seabed near Azores in the Atlantic ocean. Data was collected in a one $\mathrm{km}^{2}$ area by one meter grid points. Fig. 4 shows the time evolution of the tracking error $e=z_{s}-z-h$, control inputs and pitch angle for $t \in[600,1000]$. In this simulation the initial conditions of the vehicle and controller are $x(0)=0$, $z(0)=30, w(0)=0, \theta(0)=0, \eta_{1}(0)=[0,0,0]^{T}$ and $\eta_{2}(0)=[0,0,30]^{T}$. Seabed profile is approximated by one sinusoidal function and a bias term. The length of the window for Fourier approximation is set as $T_{x}=100 \mathrm{~m}$.

\section{Concluding Remarks}

This paper addressed the design of a seabed tracking controller for Autonomous Underwater Vehicles (AUVs). Nonlinear output regulation problem is employed to design the controller that does not require to known in advance the seabed profile and only needs one single echo sounder sensor to measure the altitude from the seabed. To cast the problem in the framework of nonlinear output regulation theory, the main steps consisted in first converting the seabed profile to a time dependent signal. Then, using Fourier series expansion, this signal is approximated by a finite number of sinusoidal signals and an exo-system is derived
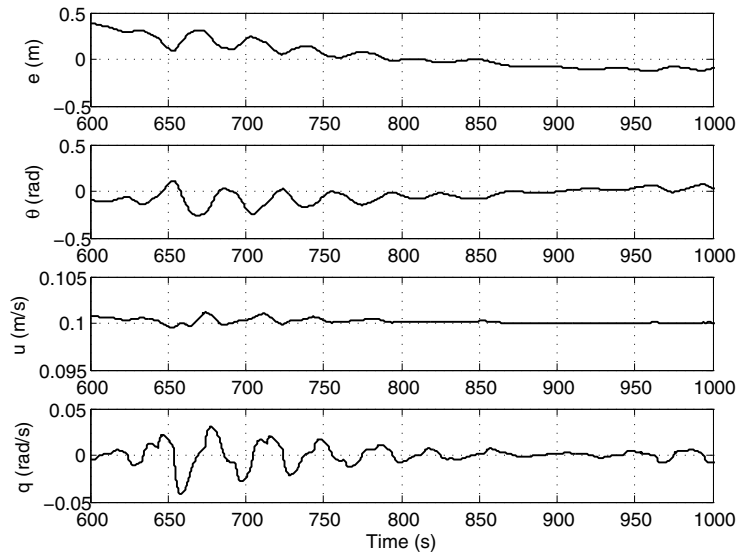

Fig. 4. Time evolution of the closed-loop signals with output feedback output regulator for a real seabed profile.

to generate this approximated seabed signal. Based on this exo-system and the plant model, full information and output feedback output regulators were designed and a solution to the regulator equation was approximated by the pseudo spectral method. Stability and zero tracking error of the closed-loop system were analysed.

The effectiveness of the new control laws was assessed in the MATLAB simulation environment with a nonlinear model of the Medusa AUV. The quality of the results obtained clearly indicate that the methodology derived achieves the seabed tracking with acceptable actuation signal. Future research will address the application of this theoretical developments to real world practical applications.

\section{REFERENCES}

[1] A. Bennett, J. Leonard, and J. Bellingham, "Bottom following for survey-class autonomous underwater vehicles," Proceedings of the ninth international Symposium on unmanned untethered submersible technology, 1995.

[2] M. Caccia, R. Bono, G. Bruzzone, and G. Veruggio, "Bottomfollowing for remotely operated vehicles," Control Engineering Practice, vol. 11, pp. 461-470, 2003.

[3] C. Silvestre, R. Cunha, N. Paulino, and A. Pascoal, "A bottomfollowing preview controller for autonomous underwater vehicles," IEEE Transaction on Control Systems Technology, vol. 17, pp. 257266, 2009.

[4] T. Fossen, Guidance and Control of Ocean Vehicles. New York: John Wiley and Sons, 1994.

[5] A. Isidori, Nonlinear Control Systems. London: Springer-Verlag, 1995.

[6] J. Huang, Nonlinear Output Regulation: Theory and applications. Philadelphia: Siam, 2004.

[7] A. Pavlov, N. van de Wouw, and H. Nijmeijer, Uniform Output Regulation Of Nonlinear Systems. Boston: Birkhauser, 2006.

[8] A. Isidori and C. I. Byrnes, "Output regulation of nonlinear systems," IEEE Transaction on Automatic Control, vol. 35, pp. 131-140, 1990.

[9] J. Huang and W. J. Rugh, "An approximation method for the nonlinear servomechanism problem," IEEE Transaction on Automatic Control, vol. 37, pp. 1395-1398, Sep. 1992.

[10] L.Marconi and L. Praly, "Uniform practical nonlinear output regulation," Automatic Control, IEEE Transactions on, vol. 53, pp. 1184$1202,2008$.

[11] D. Funaro, Polynomial Approximation of Differential Equations. Berlin, Germany: Speringer-Verlag, 1991.

[12] B. Fornberg, A Practical Guide to Pseudospectral Methods. Cambridge, UK: Cambridge university press, 1996.

[13] K. Steffens, The History of Approximation Theory: From Euler to Bernstein. Boston: Birkhauser, 2006. 